\title{
Stereotactic Brain Biopsy or Bronchoscopic/Transthoracic Needle Biopsy for Diagnosis of Metastatic Cancer Presenting Simultaneously in Lung and Brain: A Comparison of Safety and Efficacy
}

\author{
Donald A Ross ${ }^{1}$ \\ ${ }^{1}$ Oregon Health \& Science University, United States \\ Correspondence: Donald A Ross, Oregon Health \& Science University, United States. E-mail: rossdo@ohsu.edu
}

Received: August 2, 2012 Accepted: August 16, 2012 Online Published: September 19, 2012

doi:10.5539/cco.v1n2p81 URL: http://dx.doi.org/10.5539/cco.v1n2p81

\begin{abstract}
Background: When patients present with simultaneous lung and brain lesions consistent with metastases, it is often presumed that it is safer and less invasive to biopsy the lung lesion. Objective: To determine whether lung biopsy or stereotactic brain biopsy has a higher diagnostic yield and lower morbidity for tissue diagnosis in patients with simultaneous brain and lung lesions. Methods: Retrospective review of the author's stereotactic biopsy series and of the literature on brain and lung biopsies for suspected malignancy. Results: The overall diagnostic yield for bronchoscopic lung biopsy ranged from $44 \%$ to $88 \%$ and the pneumothorax rate from $1.2 \%$ to $8 \%$. No deaths were reported. The overall diagnostic yield for transthoracic lung biopsy ranged from $74 \%$ to $96 \%$ and pneumothorax rate from $2.2 \%$ to $8 \%$. No deaths were reported. The overall diagnostic yield for stereotactic brain biopsy ranged from $90.6 \%$ to $99.3 \%$ when all potential diagnoses are included. Complication rates ranged from $0.6 \%$ to $4.8 \%$ with mortality from $0 \%$ to $1.5 \%$. Several series reported no mortality. Conclusion: Stereotactic brain biopsy has a higher diagnostic yield and a lower complication rate, but a higher mortality. The inclusion of diagnoses other than metastases in the reported series may account for some of the reported mortality. When lung and brain lesions are detected simultaneously, stereotactic biopsy is a better option for tissue diagnosis.
\end{abstract}

Keywords: stereotactic biopsy, metastasis to brain, lung biopsy

\section{Introduction}

Metastasis to brain is often the first presentation of lung and other malignancies. Once the brain lesion(s) is detected, a search for the primary usually ensues. Computed tomography (CT) of the chest, abdomen, and pelvis is often the next diagnostic test. If a lesion(s) is detected in the lung, then bronchoscopic or transthoracic biopsy is usually attempted to establish a tissue diagnosis and to plan subsequent treatment (Dasgupta \& Mehta, 1999). The presumption has been that this is safer and easier than a biopsy of the brain lesion. However, the safety of stereotactic needle biopsy of brain lesions is now well established. The incision required is small and discomfort is minimal; no hair need be shaved (Sheinberg \& Ross, 1999); and some studies report that this can safely be done as an outpatient (Bhardwaj \& Bernstein, 2002; Kaakaji et al., 2001).

\section{Method}

The author reviewed his neurosurgical practice (Michigan and Oregon, USA) stereotactic biopsy series for malignant tumors of the brain and conducted a Medline literature search to compare the safety and efficacy of stereotactic biopsy with the reported safety and efficacy of bronchoscopic and transthoracic lung biopsy. The goal was to learn which is actually the safer and higher yielding procedure.

\section{Results}

From 1989 to 2009, the author conducted 161 (78 female/83 male; mean age 51.5 years) framed based or frameless stereotactic brain biopsies for malignant tumors of the brain. One hundred and forty-seven were for gliomas (106), central nervous system lymphomas (31), or pineal tumors and other rare primary central nervous system pathologies (10). Fourteen were for the tissue diagnosis of metastases to the brain. During this same time period, 150 resective procedures were performed for brain metastases. Three procedures (1.9\%), two for suspected glioma and one for primary central nervous system lymphoma were initially nondiagnostic and had to be repeated, following which one remained nondiagnostic $(0.6 \%)$. There was a single complication of a minor 
intraventricular hemorrhage following biopsy of a glioblastoma in the genu of the corpus callosum which resolved without surgery or new neurologic deficit, but prolonged the hospitalization $(0.6 \%)$. There were no new neurological deficits, no infections, and no deaths.

\section{Discussion}

\subsection{Bronchoscopic Biopsy}

There are variations in how diagnostic bronchoscopic biopsy is performed and reasons for selecting this technique over transthoracic needle biopsy. For a review of this topic, see Yung (2003). In one study, the diagnostic yield of transbronchial lung biopsy with fluoroscopic guidance was reported to be $43.8 \%$ with an incidence of pneumothorax of 1.2\% (Rittirak \& Sompradeekul, 2007). When endobronchial ultrasound, electromagnetic navigation, or both were used without fluoroscopy, diagnostic yields of $69 \%, 59 \%$, or $88 \%$ have been reported (Eberhardt, Anantham, Ernst, Feller-Kopman, \& Herth, 2007). The pneumothorax rate was 5-8\% with these procedures (Eberhardt, et al., 2007). CT guidance with virtual bronchoscopy and an ultrathin endoscope for peripheral lung lesions yielded a diagnosis in $65.4 \%$ of patients (Shinagawa et al., 2004). Thus, diagnostic yield ranged from $44 \%$ to $88 \%$ and the pneumothorax rate from $1.2 \%$ to $8 \%$. No deaths were reported.

\subsection{Transthoracic Biopsy}

For 46 patients with lesions near the chest wall, diagnostic yield of ultrasound guided transthoracic biopsy was 95.6\% with one case each of hemoptysis (2.2\%) and one pneumothorax (2.2\%) (Seyfarth et al., 2007). In another study of 91 patients undergoing ultrasound guided biopsy, the diagnostic sensitivity was $85.5 \%$ and the risk of pneumothorax 4\% (Diacon et al., 2004). CT guided biopsy in 147 cases yielded a diagnostic accuracy of $94.6 \%$ with pneumothoraces in $12.9 \%$ of which $2.7 \%$ required a chest tube (Kinoshita et al., 2006). In 506 consecutive patients, pneumothorax was detected immediately in $22.9 \%$, treated immediately in $6.5 \%$, and detected and treated in a delayed fashion in another 1.4\% (Dennie, Matzinger, Marriner, \& Maziak, 2001). In 343 biopsies performed in patients most of whom had non-diagnostic bronchoscopy, the diagnosis was made in $73.7 \%$ of malignant lesions, with pneumothorax in 7.7\%, of whom 1.6\% needed a chest tube, and hemorrhage in 1.3\% (Schneider et al., 1999). Thus, diagnostic yield ranged from $74 \%$ to $96 \%$ and pneumothorax from $2.2 \%$ to $8 \%$. No deaths were reported. Complications have been reported to be related to multiple punctures, longer intraparenchymal needle tract, and smaller lesion size (Nakatani et al., 2012; Smayra et al., 2012).

A recent economic analysis comparing CT guided needle biopsy to ultrasound guided transbronchial biopsy found the two modalities to be equivalent overall, but found that in specific cases one maybe more effective than the other (Steinfort, Liew, \& Irving, 2012).

\subsection{Stereotactic Brain Biopsy}

It is difficult to discern from the published literature on stereotactic brain biopsy how many procedures were performed for malignancies, either primary or secondary. A recent review of 290 cases of CT guided biopsy reported a diagnostic biopsy in 95.5\%, a 4.1\% incidence of symptomatic hemorrhage (two required surgery), and a mortality of $0.8 \%$ (Ersahin et al., 2011). A recent series of 134 patients done without frozen section confirmation had a diagnostic yield of 99.3\% (one targeting error) and complications in $2.2 \%$, one of which was a conservatively treated hematoma and two of which were fatal in high grade gliomas (1.5\%) (Shooman, Belli, \& Grundy, 2010). In a series of 299 biopsies by 11 surgeons for all diagnoses, diagnostic yield was $90.6 \%$ with symptomatic hemorrhage in $4.4 \%$ and death in 1.3\% (Chen et al., 2009). In another series of 622 diagnostic biopsies for all histologies, the diagnostic yield was $98.4 \%$, the overall morbidity $6.9 \%$, the symptomatic hemorrhage rate was $4.8 \%$, new, persisting neurologic deficits occurred in $1.5 \%$ and death in $1.3 \%$ (Kongkham, Knifed, Tamber, \& Bernstein, 2008). Complications were more likely in deep seated lesions and glioblastoma than other diagnoses. In 465 biopsies over a 10 year period, the diagnostic yield was $89.4 \%$ with symptomatic hemorrhage in $3.8 \%$ and mortality in 1.5\% (Dammers et al., 2008). In this series, complications were more common in frontotemporal biopsies and when the diagnosis was lymphoma.

In a series of 270 patients, a symptomatic hematoma occurred in 5\%, with a glucose level of greater than 200 highly associated with the likelihood of complications and thalamic or basal ganglia lesions at higher risk as well (McGirt et al., 2005). In a series of 355 biopsies, the diagnostic yield was $93.8 \%$ with a symptomatic hemorrhage rate of $3.6 \%$ and a mortality of $0.6 \%$, with brainstem biopsy being the only factor associated with higher morbidity (Grossman, Sadetzki, Spiegelmann, \& Ram, 2005). A series of 153 patients in whom a micro Doppler probe was used to look for vessels prior to biopsy yielded a diagnosis in 98\%, a CT detected hemorrhage in $2.5 \%$, permanent neurologic deficit in $0.6 \%$, and no mortality (Hertel, Feiden, \& Bettag, 2005). A series of 69 biopsies for inoperable lesions produced no morbidity and no mortality (Stranjalis, Protopapa, Sakas, \& Chondros, 2003). In 
two series from the same institution, 130 biopsies resulted in 3.8\% symptomatic complications ( $4 / 5$ transient) and one death $(0.8 \%)$ and 138 biopsies resulted in $2.2 \%$ symptomatic complications (one of which was a hematoma) and no deaths (Kaakaji et al., 2001). In a series of 225 patients of which $12.9 \%$ harbored metastatic tumors, biopsy was diagnostic in $95.6 \%$, major morbidity (hemorrhage or neurologic deficit) occurred in $3.6 \%$ and there was one death (0.4\%) (Sawin, Hitchon, Follett, \& Torner, 1998). Morbidity was linked to antiplatelet agents, deep seated lesion, chronic steroid use, and gliomas, but was not linked to extracranial malignancy. In a series of 122 patients by a single author (Hall, 1998), the diagnostic yield was $96 \%$ with $0.7 \%$ morbidity and one $(0.7 \%)$ fatality. Hall reviewed the published literature to date in his 1998 paper, finding 7471 reported cases with an overall mortality of $0.7 \%$, morbidity of $3.5 \%$, and diagnostic yield of $91 \%$. In another series of 500 biopsies, over 2000 specimens were obtained from 741 targets with a complication rate of $1 \%$ and mortality of $0.2 \%$ (Apuzzo, Chandrasoma, Cohen, Zee, \& Zelman, 1987). The diagnostic yield overall ranged from $89.4 \%$ to $99.3 \%$ (95.6\% overall) when all potential diagnoses were included. Non diagnostic biopsies did show necrosis in 10 cases, inflammatory response in nine cases, granuloma in one case, and gliosis in one case. On aggregate, complication rates for stereotactic brain biopsy for all pathologies ranged from $0.6 \%$ to $4.8 \%$ with mortality from $0 \%$ to $1.5 \%$. Stereotactic brain biopsy results are summarized in Table 1.

Table 1. Summary of stereotactic brain biopsy results

\begin{tabular}{lcccc}
\hline Publication (First Author, Year) & Patients (n) & Diagnostic Yield (\%) & Major Complication (\%) & Death (\%, (n)) \\
\hline Ross DA, 2012* & 161 & 99.4 & 0.6 & $0,(0)$ \\
Shooman D, 2010 & 134 & 99.3 & 2.2 & $1.5,(2)$ \\
Chen C-C, 2009 & 299 & 90.6 & 4.4 & $1.0,(3)$ \\
Kongkham PN, 2008 & 622 & 98.4 & 6.9 & $1.3,(8)$ \\
Dammers R, 2008 & 465 & 89.4 & 3.8 & $1.5,(7)$ \\
Grossman R, 2005 & 355 & 93.8 & 3.6 & $0.6,(2)$ \\
Hertel F, 2005 & 153 & 98 & 2.5 & $0(0)$ \\
Sawin PD, 1998 & 225 & 95.6 & 3.6 & $0.4,(1)$ \\
Hall WA, 1998 & 122 & 96 & 0.7 & $0.8,(1)$ \\
Apuzzo ML, 1987 & 500 & 95.6 & 1 & $0.2,(1)$ \\
\hline
\end{tabular}

*data reported here

Overall, in the published literature, when compared to lung biopsy, stereotactic brain biopsy had a higher diagnostic yield and a lower morbidity, but a higher mortality. Pneumothorax and hemoptysis were the most common complications of lung biopsy; whereas, new neurologic deficit, hematoma, or death were reported in brain biopsy, but it was not possible to discern if complications were more common in diagnoses other than metastatic tumors. In the author's hands, stereotactic biopsy had a higher yield, lower morbidity, and no mortality when compared to lung biopsy. Other authors have reported similar results (Apuzzo et al., 1987; Hertel et al., 2005; Shooman et al., 2010; Stranjalis et al., 2003). It is likely that the wide range of pathologies included in the other published series of stereotactic brain biopsy may contribute to the morbidity of these procedures. Glioblastomas (Kongkham et al., 2008; Sawin et al., 1998) and lymphomas (Dammers et al., 2008) have been reported as more likely to bleed when biopsied. Targets located adjacent to the ventricles or the Sylvian fissure may be especially challenging and biopsy of vascular anomalies or infarcts may be disastrous (Neal \& Apuzzo, 1989). Since many metastatic tumors to the brain are located in the gray-white junction of the hemispheres, the thalamus, or the cerebellum and present straightforward targets for stereotactic biopsy, it may be that the morbidity of these procedures is lower than the overall reported morbidity for stereotactic biopsy, but this data cannot be gleaned from the literature.

In order to be less morbid than lung biopsy, stereotactic brain biopsy should be performed with no mortality and with rare serious complications. If these criteria can be met, whenever the lung mass is thought to be technically suboptimal for biopsy or if the brain mass is in an accessible location for stereotactic biopsy, the brain lesion should be considered for biopsy in preference to the lung lesion.

\section{Acknowledgements}


The author thanks Shirley McCarteny, Ph.D., for editorial assistance.

\section{References}

Apuzzo, M. L., Chandrasoma, P. T., Cohen, D., Zee, C. S., \& Zelman, V. (1987). Computed imaging stereotaxy: experience and perspective related to 500 procedures applied to brain masses. Neurosurgery, 20(6), 930-937. http://journals.lww.com/neurosurgery/Abstract/1987/06000/Computed_Imaging_Stereotaxy_Experience_a nd.19.aspx

Bhardwaj, R. D., \& Bernstein, M. (2002). Prospective feasibility study of outpatient stereotactic brain lesion biopsy. Neurosurgery, 51(2), 358-361. http://dx.doi.org/10.1227/01.NEU.0000020572.00872.F1

Chen, C-C., Hsu, P-W., Erich Wu, T-W., Lee, S-T., Chang, C-N., Wei, K.-C., ... Huang, Y-C. (2009). Stereotactic brain biopsy: Single center retrospective analysis of complications. Clinical Neurology \& Neurosurgery, 111(10), 835-839. http://dx.doi.org/10.1016/j.clineuro.2009.08.013

Dammers, R., Haitsma, I. K., Schouten, J. W., Kros, J. M., Avezaat, C. J. J., \& Vincent, A. J. P. E. (2008). Safety and efficacy of frameless and frame-based intracranial biopsy techniques. Acta Neurochirurgica, 150(1), 23-29. http://dx.doi.org/10.1007/s00701-007-1473-x

Dasgupta, A., \& Mehta, A. C. (1999). Transbronchial needle aspiration. An underused diagnostic technique. Clinics in Chest Medicine, 20(1), 39-51. http://dx.doi.org/10.1016/S0272-5231(05)70125-8

Dennie, C. J., Matzinger, F. R., Marriner, J. R., \& Maziak, D. E. (2001). Transthoracic needle biopsy of the lung: results of early discharge in 506 outpatients. Radiology, 219(1), 247-251. http://radiology.rsna.org/content/219/1/247.long

Diacon, A. H., Schuurmans, M. M., Theron, J., Schubert, P. T., Wright, C. A., \& Bolliger, C. T. (2004). Safety and yield of ultrasound-assisted transthoracic biopsy performed by pulmonologists. Respiration, 71(5), 519-522. http://dx.doi.org/10.1159/000080638

Eberhardt, R., Anantham, D., Ernst, A., Feller-Kopman, D., \& Herth, F. (2007). Multimodality bronchoscopic diagnosis of peripheral lung lesions: a randomized controlled trial. American Journal of Respiratory \& Critical Care Medicine, 176(1), 36-41. http://dx.doi.org/10.1164/rccm.200612-1866OC

Ersahin, M., Karaaslan, N., Gurbuz, M. S., Hakan, T., Berkman, M. Z., Ekinci, O., ... Aker, F. V. (2011). The safety and diagnostic value of frame-based and CT-guided stereotactic brain biopsy technique. Turkish neurosurgery, 21(4), 582-590. http://dx.doi.org/10.5137/1019-5149.JTN .4656-11.1

Grossman, R., Sadetzki, S., Spiegelmann, R., \& Ram, Z. (2005). Haemorrhagic complications and the incidence of asymptomatic bleeding associated with stereotactic brain biopsies. Acta Neurochirurgica, 147(6), 627-631. http://dx.doi.org/10.1007/s00701-005-0495-5

Hall, W. A. (1998). The safety and efficacy of stereotactic biopsy for intracranial lesions. Cancer, 82(9), 1749-1755.

Hertel, F., Feiden, W., \& Bettag, M. (2005). The value of micro-Doppler in stereotactic brain biopsy. Minimally Invasive Neurosurgery, 48(3), 165-168. http://dx.doi.org/10.1055/s-2004-830269

Kaakaji, W., Barnett, G. H., Bernhard, D., Warbel, A., Valaitis, K., \& Stamp, S. (2001). Clinical and economic consequences of early discharge of patients following supratentorial stereotactic brain biopsy. Journal of Neurosurgery, 94(6), 892-898. http://thejns.org/doi/abs/10.3171/jns.2001.94.6.0892

Kinoshita, F., Kato, T., Sugiura, K., Nishimura, M., Kinoshita, T., Hashimoto, M., ... Ogawa, T. (2006). CT-guided transthoracic needle biopsy using a puncture site-down positioning technique. American Journal of Roentgenology, 187(4), 926-932. http://dx.doi.org/10.2214/AJR.05.0226

Kongkham, P. N., Knifed, E., Tamber, M. S., \& Bernstein, M.. (2008). Complications in 622 cases of frame-based stereotactic biopsy, a decreasing procedure. Canadian Journal of Neurological Sciences, 35(1), 79-84. http://cjns.metapress.com/content/r844353560371l85/

McGirt, M. J., Woodworth, G. F., Coon, A. L., Frazier, J. M., Amundson, E., Garonzik, I., ... Weingart, J. D. (2005). Independent predictors of morbidity after image-guided stereotactic brain biopsy: a risk assessment of 270 cases. Journal of Neurosurgery, 102(5), 897-901. http://dx.doi.org/10.3171/jns.2005.102.5.0897

Nakatani, M., Tanigawa, N., Kariya, S., Komemushi, A., Yagi, R., \& Sawada, S. (2012). Analysis of factors influencing accuracy and complications in CT-guided lung biopsy. Minimally invasive therapy \& allied technologies: MITAT: official journal of the Society for Minimally Invasive Therapy. 
http://dx.doi.org/10.3109/13645706.2012.662155

Neal, J. H., \& Apuzzo, M. L. J. (1989). Experience with the BRW Stereotactic System. In Para Chandrasoma \& Michael L. J. Apuzzo (Eds.), Stereotactic brain biopsy (pp. 23-43). New York: Igaku-Shoin.

Rittirak, W., \& Sompradeekul, S. (2007). Diagnostic yield of fluoroscopy-guided transbronchial lung biopsy in non-endobronchial lung lesion. Journal of the Medical Association of Thailand, 90(2), 68-73.

Sawin, P. D., Hitchon, P. W., Follett, K. A., \& Torner, J. C. (1998). Computed imaging-assisted stereotactic brain biopsy: a risk analysis of 225 consecutive cases. Surgical Neurology, 49(6), 640-649. http://dx.doi.org/10.1016/S0090-3019(97)00435-7

Schneider, C. P., Proschild, A., Mader, I., Treutler, D., Leonhardi, J., \& Wiesner, B. (1999). CT-assisted transthoracic puncture of pulmonary lesions and mediastinal infiltrations. A retrospective study of 300 patients. Pneumologie, 53(2), 71-76.

Seyfarth, H. J., Wirtz, H., Borte, G., Gradistanac, T., Gessner, C., \& Hammerschmidt, S. (2007). Ultrasound guided transthoracic biopsy - safety and efficaces. Pneumologie, 61(9), 563-567. http://dx.doi.org/10.1055/s-2007-980056

Sheinberg, M. A., \& Ross, D. A. (1999). Cranial procedures without hair removal. Neurosurgery, 44(6), 1263-1265; discussion 1265-1266. http://journals.lww.com/neurosurgery/Abstract/1999/06000/Cranial_ Procedures_without_Hair_Removal.54.aspx

Shinagawa, N., Yamazaki, K., Onodera, Y., Miyasaka, K., Kikuchi, E., Dosaka-Akita, H., \& Nishimura, M. (2004). CT-guided transbronchial biopsy using an ultrathin bronchoscope with virtual bronchoscopic navigation. Chest, 125(3), 1138-1143. http://dx.doi.org/10.1378/chest.125.3.1138

Shooman, D., Belli, A., \& Grundy, P. L. (2010). Image-guided frameless stereotactic biopsy without intraoperative neuropathological examination. Journal of Neurosurgery, 113(2), 170-178. http://dx.doi.org/10.3171/2009. 12.JNS09573

Smayra, T., Braidy, C., Menassa-Moussa, L., Hlais, S., Haddad-Zebouni, S., \& Aoun, N. (2012). Risk factors of pneumothorax and hemorrhage in lung biopsy: a single institution experience. J Med Liban, 60(1), 4-13.

Steinfort, D. P., Liew, D., \& Irving, L. B. (2012). Radial probe ebus versus ct-guided needle biopsy for evaluation of peripheral pulmonary lesions: an economic analysis. The European respiratory journal: official journal of the European Society for Clinical Respiratory Physiology. http://dx.doi.org/10.1183/09031936.00044612

Stranjalis, G., Protopapa, D., Sakas, D. E., \& Chondros, D. (2003). Stereotactic biopsy in the era of advanced neuroimaging. Does the minimal therapeutic gain justify its current wide use? Minimally Invasive Neurosurgery, 46(2), 90-93. http://dx.doi.org/10.1055/s-2003-39346

Yung, Rex C. W. (2003). Tissue diagnosis of suspected lung cancer: selecting between bronchoscopy, transthoracic needle aspiration, and resectional biopsy. Respiratory Care Clinics of North America, 9(1), 51-76. 